\title{
Solvability of integral boundary value problems at resonance in $R^{n}$
}

\author{
Shiying Song ${ }^{1}$, Shuman Meng ${ }^{1}$ and Yujun Cui ${ }^{2^{*}}$ (B)
}

\author{
"Correspondence: \\ cyj720201@163.com \\ ${ }^{2}$ State Key Laboratory of Mining \\ Disaster Prevention and Control \\ Co-founded by Shandong Province \\ and the Ministry of Science and \\ Technology, Shandong University of \\ Science and Technology, Qingdao, \\ P.R. China \\ Full list of author information is \\ available at the end of the article
}

\begin{abstract}
Under a resonance condition involving integral boundary value problems for a second-order nonlinear differential equation in $\mathbb{R}^{n}$, we show its solvability by using the coincidence degree theory of Mawhin and the theory of matrix diagonalization in linear algebra.
\end{abstract}

MSC: 34B15

Keywords: Coincidence degree theorem; Integral boundary conditions; Resonance

\section{Introduction}

Let $A=\left(a_{i j}\right)_{n \times n}$ be a square matrix of order $n . \alpha:[0,1] \rightarrow \mathbb{R}$ is a bounded variation function, and $\int_{0}^{1} u(t) d \alpha(t)=\left(\int_{0}^{1} u_{1}(t) d \alpha(t), \int_{0}^{1} u_{2}(t) d \alpha(t), \ldots, \int_{0}^{1} u_{n}(t) d \alpha(t)\right)^{T}$ where $\int_{0}^{1} u_{i}(t) d \alpha(t)$ denotes the Riemann-Stieltjes integrals of $u_{i}$ with respect to $\alpha$ and $u^{T}$ denotes the vector transpose of the row vector $u$. Take $h=\int_{0}^{1} t d \alpha(t)$ and $B=h A$.

In this paper, we will study the existence of solutions for the following integral boundary value problem at resonance in $\mathbb{R}^{n}$ :

$$
\left\{\begin{array}{l}
-u^{\prime \prime}(t)=f\left(t, u(t), u^{\prime}(t)\right), \quad t \in(0,1), \\
u(0)=0, \quad u(1)=A \int_{0}^{1} u(t) d \alpha(t),
\end{array}\right.
$$

under the following assumptions:

(H1) $B$ is a diagonalization matrix, and $\operatorname{det}(I-B)=0$;

(H2) $\int_{0}^{1} t(1-t) d \alpha(t) \neq 0$;

(H3) $f:[0,1] \times \mathbb{R}^{2 n} \rightarrow \mathbb{R}^{n}$ satisfies the Carathéodory conditions.

If the condition $\left(H_{1}\right)$ is considered, the associated linear problem $-u^{\prime \prime}(t)=0, u(0)=0$, $u(1)=A \int_{0}^{1} u(t) d \alpha(t)$ has a nontrivial solution $u(t)=\psi t$ with $\psi \in \operatorname{Ker}(I-B)$. This means that this problem is a resonant integral boundary value problem (IBVP).

Integral boundary value problems of this form arise in different areas of applied mathematics and physics such as heat conduction, thermoelasticity, underground water flow, and plasma physics. Moreover, integral boundary value problems constitute a very important class of problems based on the fact that two-point, three-point, multi-point and nonlocal boundary value problems can be treated as special cases of Riemann-Stieltjes integral boundary value problems. As a result, the existence of solutions for such problems has received great attention (see $[1,5-9,11,12,14,15,23,26-28])$. It is well known

(c) The Author(s) 2019. This article is distributed under the terms of the Creative Commons Attribution 4.0 International License (http://creativecommons.org/licenses/by/4.0/), which permits unrestricted use, distribution, and reproduction in any medium, provided you give appropriate credit to the original author(s) and the source, provide a link to the Creative Commons license, and indicate if changes were made. 
that, when $n=1$, the existence theory of integral boundary value problems for ordinary differential equations or fractional differential equations has been well studied; we refer the reader to $[4,10,17,20,21,24,25,29-35,37]$ for some recent results at non-resonance and to $[2,3,16,18,22,23,27,36]$ for results at resonance. When $n \geq 2$ and $A$ is not a diagonal matrix, IBVP (1.1) becomes a system of ordinary differential equations with coupled boundary conditions. Differential systems with coupled integral boundary conditions can be applied to reaction-diffusion phenomena, interaction problems and Lotka-Volterra models. Recently, there have been many papers addressing the existence of solutions for differential systems of coupled integral boundary value problems; see, for example, $[1,3$, 5-7, 9, 11-14].

To the best of our knowledge, the solvability of problem (1.1) at resonance has not been considered before. The main purpose of this paper is to establish an existence result for problem (1.1) when $n \geq 2$. Our main method is based on the coincidence degree theory of Mawhin and the theory of matrix diagonalization in linear algebra.

We end this section by recalling some notations and abstract results from coincidence degree theory.

Let $X$ and $Y$ be two real Banach spaces, $L: \operatorname{dom} L \subset X \rightarrow Y$ be a linear Fredholm operator of index zero, and $P: X \rightarrow X$ and $Q: Y \rightarrow Y$ be two continuous projectors such that

$$
\operatorname{Im} P=\operatorname{ker} L, \quad \operatorname{ker} Q=\operatorname{Im} L, \quad X=\operatorname{ker} L \oplus \operatorname{ker} P, \quad Y=\operatorname{Im} L \oplus \operatorname{Im} Q .
$$

It follows from the above equalities that the reduced operator

$$
\left.L\right|_{\operatorname{dom} L \cap \operatorname{Ker} P}: \operatorname{dom} L \cap \operatorname{ker} P \rightarrow \operatorname{Im} L
$$

is invertible. We denote its inverse by $K_{P}$ (the generalized inverse operator of $L$ ). If $\Omega$ is an open bounded subset of $X$ such that $\operatorname{dom} L \cap \Omega \neq \emptyset$, the mapping $N: X \rightarrow Y$ will be called $L$-compact on $\bar{\Omega}$ if $Q N(\bar{\Omega})$ is bounded and $K_{P}(I-Q) N: \bar{\Omega} \rightarrow X$ is compact.

We make use of the following result from Mawhin [19].

Theorem 1.1 ([19] (Mawhin continuation theorem)) Let $L: \operatorname{dom} L \subset X \rightarrow Y$ be a Fredholm operator of index zero and $N$ be L-compact on $\bar{\Omega}$. The equation $L \varphi=N \varphi$ has at least one solution in $\operatorname{dom} L \cap \bar{\Omega}$ if the following conditions are satisfied:

(1) $L \varphi \neq \lambda N \varphi$ for every $(\varphi, \lambda) \in[(\operatorname{dom} L \backslash \operatorname{ker} L) \cap \partial \Omega] \times(0,1)$;

(2) $N \varphi \notin \operatorname{Im} L$ for every $\varphi \in \operatorname{ker} L \cap \partial \Omega$;

(3) $\operatorname{deg}\left(\left.J Q N\right|_{\operatorname{ker} L}, \Omega \cap \operatorname{ker} L, 0\right) \neq 0$, where $J: \operatorname{Im} Q \rightarrow \operatorname{Ker} L$ is some isomorphism.

\section{Preliminaries}

We use the classical spaces $X=C^{1}\left([0,1], \mathbb{R}^{n}\right)$ and $Y=L^{1}\left([0,1], \mathbb{R}^{n}\right)$. For $u \in X$, we use the norm $\|u\|_{X}=\max \left\{\|u\|_{\infty},\left\|u^{\prime}\right\|_{\infty}\right\}$, where $\|u\|_{\infty}=\max _{t \in[0,1]}\left\{\left|u_{1}(t)\right|,\left|u_{2}(t)\right|, \ldots,\left|u_{n}(t)\right|\right\}$, and denote the norm in $L^{1}\left([0,1], \mathbb{R}^{n}\right)$ by $\|u\|_{1}=\max _{1 \leq i \leq n} \int_{0}^{1}\left|u_{i}(t)\right| d t$. We also use the Sobolev space defined by

$$
X_{0}=\left\{u \in X: u^{\prime} \text { is absolutely continuous on }[0,1] \text { and } u^{\prime \prime} \in Y\right\} \text {. }
$$


We define $L$ to be the linear operator from $D(L) \subset X$ to $Y$ with

$$
\operatorname{dom} L=\left\{u \in X_{0}: u(0)=0, u(1)=A \int_{0}^{1} u(t) d \alpha(t)\right\}
$$

and for $u \in D(L), L u=-u^{\prime \prime}$. Let $N: X \rightarrow Y$ be the nonlinear operator defined by

$$
(N u)(t)=f\left(t, u(t), u^{\prime}(t)\right), \quad t \in[0,1]
$$

Thus, problem (1.1) can be written as $L u=N u$.

Lemma 2.1 The following results hold:

(1) $\operatorname{Ker} L=\left\{u \in X: u(t)=\psi t, \psi \in \operatorname{Ker}(I-B) \subset \mathbb{R}^{n}\right\}$.

(2) $\operatorname{Im} L=\{v \in Y: \varphi(v) \in \operatorname{Im}(I-B)\}$, where $\varphi: Y \rightarrow \mathbb{R}^{n}$ is a linear operator defined by

$$
\varphi(v)=A \int_{0}^{1} \int_{0}^{1} G(t, s) v(s) d s d \alpha(t)
$$

where

$$
G(t, s)= \begin{cases}t(1-s), & 0 \leq t \leq s \leq 1 \\ s(1-t), & 0 \leq s \leq t \leq 1\end{cases}
$$

Proof (1) For $u \in \operatorname{Ker} L$, we obtain $-u^{\prime \prime}=0$. Then $u(t)=\psi t+\psi_{1}$ with $\psi, \psi_{1} \in \mathbb{R}^{n}$. With consideration of the boundary conditions $u(0)=0$ and $u(1)=A \int_{0}^{1} u(t) d \alpha(t)$, we conclude that $\psi_{1}=0$ and $\psi=A \int_{0}^{1} \psi t d \alpha(t)=A(h \psi)=B \psi$. Thus, $\psi \in \operatorname{Ker}(I-B)$. Again, if $u(t)=\psi t$ with $\psi \in \operatorname{Ker}(I-B)$, then $u \in \operatorname{Ker} L$.

(2) For $v \in \operatorname{Im} L$, there exists $u \in \operatorname{dom} L$ such that $-u^{\prime \prime}(t)=v(t)$. Thus,

$$
u(t)=u(0)(1-t)+u(1) t+\int_{0}^{1} G(t, s) v(s) d s
$$

Using the boundary conditions $u(0)=0$ and $u(1)=A \int_{0}^{1} u(t) d \alpha(t)$, it follows from (2.3) that

$$
\begin{aligned}
u(1) & =A \int_{0}^{1} u(t) d \alpha(t)=A \int_{0}^{1}\left(u(1) t+\int_{0}^{1} G(t, s) v(s) d s\right) d \alpha(t) \\
& =A(h u(1))+\varphi(v)=B u(1)+\varphi(v) .
\end{aligned}
$$

This implies that $\varphi(v)=(I-B) u(1)$. Thus, $v \in\{v \in Y: \varphi(v) \in \operatorname{Im}(I-B)\}$. Conversely, if $v \in Y$ and $\varphi(v) \in \operatorname{Im}(I-B)$, let

$$
u(t)=\xi t+\int_{0}^{1} G(t, s) v(s) d s
$$

where $\xi \in \mathbb{R}^{n}$ satisfies

$$
(I-B) \xi=\varphi(v)
$$


Then $-u^{\prime \prime}(t)=v(t), u(0)=0, u(1)=\xi$ and

$$
A \int_{0}^{1} u(t) d \alpha(t)=A \xi \int_{0}^{1} t d \alpha(t)+\varphi(v)=B \xi+\varphi(v)=\xi
$$

Thus, $u(1)=A \int_{0}^{1} u(t) d \alpha(t)$ and $v \in \operatorname{Im} L$. This completes the proof.

Recall that a matrix is diagonalizable over the field $\mathbb{R}$ if and only if its minimal polynomial is a product of distinct linear factors over $\mathbb{R}$. Thus, it follows from $\left(H_{1}\right)$ that the minimal polynomial of the matrix $B$ can be written as

$$
\mu_{B}(x)=(1-x) g(x)
$$

where $1-x$ and $g(x)$ are two polynomials which are relatively prime. Hence, there exist two polynomials, $a(t)$ and $b(t)$, such that

$$
(1-x) a(x)+g(x) b(x)=1
$$

From this, we conclude that $(I-B) a(B)+g(B) b(B)=I$. Thus,

$$
\begin{aligned}
& \operatorname{Im}(I-B)=\operatorname{Ker} g(B), \\
& \operatorname{Ker}(I-B)=\operatorname{Im} g(B), \\
& \mathbb{R}^{n}=\operatorname{Im}(I-B) \oplus \operatorname{Im} g(B) .
\end{aligned}
$$

Moreover, by (2.5), we have $(I-B) g(B)=0$, that is,

$$
g(B) B=g(B)
$$

Consequently, we deduce that

$$
g(B) g(B)=g(1) g(B)
$$

where $g(1) \neq 0$ holds following from the fact that $1-x$ and $g(x)$ are two relatively prime polynomials.

Example 2.1 When $B^{m}=B$ with $2 \leq m \leq n$, the minimal polynomial of the matrix, $B$, is

$$
\mu_{B}(x)=x-x^{m}=(1-x)\left(x+x^{2}+\cdots+x^{m-1}\right)=(1-x) g(x)
$$

Thus, we have

$$
g(1)=m-1 \text {. }
$$

When $B^{m}=I$ with a $2 \leq m \leq n$, the minimal polynomial of the matrix, $B$, can be explicitly given by

$$
\mu_{B}(x)=1-x^{m}=(1-x)\left(1+x+x^{2}+\cdots+x^{m-1}\right)=(1-x) g(x) .
$$


Thus, we obtain

$$
g(1)=m
$$

Lemma 2.2 $L$ is a Fredholm operator of index zero.

Proof We define an operator $Q: Y \rightarrow Y$ by

$$
(Q v)(t)=k g(B) \varphi(v), \quad v \in Y
$$

where $\varphi$ is given in (2.1) and $k=\frac{2 h}{g(1) \int_{0}^{1} t(1-t) d \alpha(t)}$. Note that if $w(t)=\psi$ with $\psi \in \mathbb{R}^{n}$, we have

$$
\begin{aligned}
(Q w)(t) & =k g(B) A \psi \int_{0}^{1} \int_{0}^{1} G(t, s) d s d \alpha(t) \\
& =k g(B) A \psi \int_{0}^{1} \frac{t(1-t)}{2} d \alpha(t)=\frac{h}{g(1)} g(B) A \psi \\
& =\frac{1}{g(1)} g(B) B \psi=\frac{1}{g(1)} g(B) \psi
\end{aligned}
$$

Hence,

$$
\begin{aligned}
\left(Q^{2} v\right)(t) & =\frac{1}{g(1)} g(B)(k g(B) \varphi(v)) \\
& =\frac{k}{g(1)} g(B) g(B) \varphi(v) \\
& =k g(B) \varphi(v)=(Q v)(t) .
\end{aligned}
$$

Therefore, the map $Q$ is a continuous linear projector. Moreover, by (2.6) and Lemma 2.1, we have

$$
v \in \operatorname{Ker} Q \quad \Leftrightarrow \quad \varphi(v) \in \operatorname{Ker} g(B) \quad \Leftrightarrow \quad \varphi(v) \in \operatorname{Im}(I-B) \quad \Leftrightarrow \quad v \in \operatorname{Im} L .
$$

This means that $\operatorname{Ker} Q=\operatorname{Im} L$. For $v \in Y, v-Q v \in \operatorname{Ker} Q=\operatorname{Im} L$. Therefore, $Y=\operatorname{Im} L+\operatorname{Im} Q$, and, again, $\operatorname{Im} L \cap \operatorname{Im} Q=\{0\}$. Hence, $Y=\operatorname{Im} L \oplus \operatorname{Im} Q$. Combining the previous results with the additional information that $\operatorname{Im} L$ is closed, we conclude that $L$ is a Fredholm operator of index zero.

In what follows, we make the following assumption on the matrix $B$ :

(H4) There exists $l \in \mathbb{R}$ such that $l(I-B)(I-B)=(I-B)$.

Example 2.2 When $B^{2}=B$, we can take $l=1$ such that

$$
(I-B)(I-B)=I-2 B+B^{2}=I-B .
$$

If $B^{2}=I$, we can take $l=\frac{1}{2}$ such that

$$
l(I-B)(I-B)=\frac{1}{2}\left(I-2 B+B^{2}\right)=\frac{1}{2}(2 I-2 B)=I-B .
$$


Lemma 2.3 Assuming that (H4) holds,

$$
l(I-B) \psi=\psi, \quad \forall \psi \in \operatorname{Im}(I-B) .
$$

Proof Let $\psi \in \operatorname{Im}(I-B)$ so that $\psi=(I-B) \psi_{1}$ for $\psi_{1} \in \mathbb{R}^{n}$. Using the condition (H4), we have

$$
l(I-B) \psi=l(I-B)(I-B) \psi_{1}=(I-B) \psi_{1}=\psi .
$$

Lemma 2.4 If $\Omega$ is an open bounded subset such that $\operatorname{dom} L \cap \Omega \neq \emptyset$, then $N$ is $L$-compact on $\bar{\Omega}$.

Proof Define the linear operator $P: X \rightarrow X$ by

$$
(P u)(t)=\frac{1}{g(1)} g(B) u(1) t
$$

Then we have

$$
\begin{aligned}
\left(P^{2} u\right)(t) & =\frac{1}{g(1)} g(B)\left(\frac{1}{g(1)} g(B) u(1)\right) t \\
& =\frac{1}{g^{2}(1)} g(B) g(B) u(1) t=\frac{1}{g(1)} g(B) u(1) t=(P u)(t) .
\end{aligned}
$$

This shows that $P$ is a continuous projection operator. In the following, we will assert that $\operatorname{Im} P=\operatorname{Ker} L$. In fact, if $v \in \operatorname{Im} P$, there is $u \in X$, such that

$$
v(t)=P u(t)=\frac{1}{g(1)} g(B) u(1) t
$$

Thus, it follows from (2.6) that $v \in \operatorname{Ker} L$. Conversely, if $v \in \operatorname{Ker} L$, we have

$$
v(t)=\psi t, \quad \psi \in \operatorname{Ker}(I-B)
$$

On account of the second identity in (2.6), there exists $\psi_{1} \in \mathbb{R}^{n}$, such that $\psi=g(B) \psi_{1}$. Taking $u(t)=g(1) \psi_{1}$, we then have

$$
P u(t)=\frac{1}{g(1)} g(B) u(1) t=g(B) \psi_{1} t=\psi t,
$$

which implies that $v \in \operatorname{Im} P$. Thus, we conclude that $\operatorname{Im} P=\operatorname{Ker} L$, and consequently,

$$
X=\operatorname{Ker} L \oplus \operatorname{Ker} P .
$$

Therefore, the generalized inverse $K_{P}: \operatorname{Im} L \rightarrow \operatorname{dom} L \cap \operatorname{Ker} P$ can be given by

$$
\left(K_{P} v\right)(t)=\int_{0}^{1} G(t, s) v(s) d s+l\left(I-\frac{1}{g(1)} g(B)\right) \varphi(v) t,
$$


where the constant $l$ is given in (H4). Note that, since $G(1, s)=0$ for all $s \in[0,1]$ and from (2.9), we have

$$
P\left(K_{P} v\right)(t)=\frac{l}{g(1)} g(B)\left(I-\frac{1}{g(1)} g(B)\right) \varphi(v) t=0
$$

Hence, $K_{P} v \in \operatorname{Ker} P$. For $v \in \operatorname{Im} L$, we know that

$$
\left(K_{P} v\right)(1)=l\left(I-\frac{1}{g(1)} g(B)\right) \varphi(v)
$$

and

$$
\begin{aligned}
A & \int_{0}^{1}\left(K_{P} v\right)(t) d \alpha(t) \\
& =A \int_{0}^{1}\left(\int_{0}^{1} G(t, s) v(s) d s+l\left(I-\frac{1}{g(1)} g(B)\right) \varphi(v) t\right) d \alpha(t) \\
& =\varphi(v)+l A\left(I-\frac{1}{g(1)} g(B)\right) \varphi(v) \int_{0}^{1} t d \alpha(t) \\
& =\varphi(v)+l A\left(I-\frac{1}{g(1)} g(B)\right) \varphi(v) h=\varphi(v)+l B\left(I-\frac{1}{g(1)} g(B)\right) \varphi(v) \\
& =\varphi(v)+l(B-I+I)\left(I-\frac{1}{g(1)} g(B)\right) \varphi(v) \\
& =\varphi(v)-l(I-B)\left(I-\frac{1}{g(1)} g(B)\right) \varphi(v)+l\left(I-\frac{1}{g(1)} g(B)\right) \varphi(v) \\
& =\varphi(v)-l(I-B) \varphi(v)+l\left(I-\frac{1}{g(1)} g(B)\right) \varphi(v)=l\left(I-\frac{1}{g(1)} g(B)\right) \varphi(v) .
\end{aligned}
$$

Therefore, $\left(K_{P} v\right)(1)=A \int_{0}^{1}\left(K_{P} v\right)(t) d \alpha(t)$, and consequently, $K_{P}$ is well defined. Furthermore, if $u \in \operatorname{dom} L \cap \operatorname{Ker} P$, then, using (2.7) and Lemma 2.3, we have

$$
\begin{aligned}
& \left(K_{P} L u\right)(t) \\
& \quad=-\int_{0}^{1} G(t, s) u^{\prime \prime}(s) d s+l\left(I-\frac{1}{g(1)} g(B)\right) \varphi(L u) t \\
& =u(t)-u(1) t+l\left(I-\frac{1}{g(1)} g(B)\right) A\left(\int_{0}^{1}(u(t)-u(1) t) d \alpha(t)\right) t \\
& =u(t)-u(1) t+l\left(I-\frac{1}{g(1)} g(B)\right)\left(A \int_{0}^{1} u(t) d \alpha(t)-A h u(1)\right) t \\
& =u(t)-u(1) t+l\left(I-\frac{1}{g(1)} g(B)\right)(I-B) u(1) t \\
& =u(t)-u(1) t+l(I-B) u(1) t \\
& =u(t) .
\end{aligned}
$$


This shows that $K_{P}=\left(\left.L\right|_{\operatorname{dom} L \cap \operatorname{Ker} P}\right)^{-1}$ and that $L K_{P} v(t)=v(t), v \in \operatorname{Im} L$. For $v \in \operatorname{dom} L$, by (2.8) we obtain

$$
\left(K_{P} v\right)^{\prime}(t)=\int_{t}^{1}(1-s) v(s) d s-\int_{0}^{t} s v(s) d s+l\left(I-\frac{1}{g(1)} g(B)\right) A \int_{0}^{1} \int_{0}^{1} G(t, s) v(s) d s d \alpha(t) .
$$

Notice that

$$
\begin{aligned}
\max _{t \in[0,1]}\left|\int_{0}^{1} G(t, s) v(s) d s\right| & \leq \max _{t \in[0,1]} \int_{0}^{1} t(1-t)|v(s)| d s \leq \frac{\|v\|_{1}}{4} \\
\max _{t \in[0,1]}\left|\int_{0}^{1} G_{t}^{\prime}(t, s) v(s) d s\right| & =\max _{t \in[0,1]}\left|-\int_{0}^{t} s v(s) d s+\int_{t}^{1}(1-s) v(s) d s\right| \\
& \leq \max _{t \in[0,1]}\left(t \int_{0}^{t}|v(s)| d s+(1-t) \int_{t}^{1}|v(s)| d s\right) \\
& \leq \max _{t \in[0,1]}\left(t \int_{0}^{1}|v(s)| d s+(1-t) \int_{0}^{1}|v(s)| d s\right)=\|v\|_{1},
\end{aligned}
$$

and

$$
\begin{aligned}
\left|\int_{0}^{1} \int_{0}^{1} G(t, s) v(s) d s d \alpha(t)\right| & \leq \int_{0}^{1} \int_{0}^{1} t(1-t)|v(s)| d s d\left(\bigvee_{0}^{t}(\alpha)\right) \\
& =\int_{0}^{1} t(1-t) d\left(\bigvee_{0}^{t}(\alpha)\right) \cdot\|v\|_{1},
\end{aligned}
$$

where $\bigvee_{0}^{t}(\alpha)$ denotes the total variation of $\alpha$ on $[0, t]$ defined by

$$
\bigvee_{0}^{t}(\alpha)=\sup _{\mathcal{P}} \sum_{i=1}^{n}\left|\alpha\left(t_{i}\right)-\alpha\left(t_{i-1}\right)\right|
$$

where the supremum runs over the set of all partitions

$$
\mathcal{P}=\left\{P=\left\{t_{0}, \ldots, t_{n}\right\} \mid P \text { is a partition of }[0, t]\right\}
$$

Let $\|\cdot\|_{*}$ be the max-norm of matrices defined by

$$
\|A\|_{*}=\max _{1 \leq i \leq n, 1 \leq j \leq m}\left|a_{i j}\right|, \quad \text { for } A=\left(a_{i j}\right)_{n \times m}
$$

and $\|\cdot\|_{\mathbb{R}^{n}}$ be the maximum norm in $\mathbb{R}^{n}$. Then we have

$$
\|A v\|_{\mathbb{R}^{n}} \leq m\|A\|_{*}\|v\|_{\mathbb{R}^{m}}, \quad \text { for } A=\left(a_{i j}\right)_{n \times m} \text { and } v \in \mathbb{R}^{m} \text {. }
$$

Thus,

$$
\left\|K_{P} v\right\|_{\infty} \leq\left(\frac{1}{4}+n|l| \cdot\left\|\left(I-\frac{1}{g(1)} g(B)\right) A\right\|_{*}^{1} \int_{0}^{1} t(1-t) d\left(\bigvee_{0}^{t}(\alpha)\right)\right)\|v\|_{1}
$$


and

$$
\left\|K_{P}^{\prime} v\right\|_{\infty} \leq\left(1+n|l| \cdot\left\|\left(I-\frac{1}{g(1)} g(B)\right) A\right\|_{*} \int_{0}^{1} t(1-t) d\left(\bigvee_{0}^{t}(\alpha)\right)\right)\|v\|_{1}
$$

Consequently, we conclude that

$$
\left\|K_{P} v\right\|_{X} \leq M\|v\|_{1}
$$

where $M=1+n|l|\left\|\left(I-\frac{1}{g(1)} g(B)\right) A\right\|_{*} \int_{0}^{1} t(1-t) d\left(\bigvee_{0}^{t}(\alpha)\right)$. It is easy to see that

$$
(Q N u)(t)=k g(B) A \int_{0}^{1} \int_{0}^{1} G(t, s) f\left(s, u(s), u^{\prime}(s)\right) d s d \alpha(t) \in \mathbb{R}^{n}
$$

and

$$
\begin{aligned}
& K_{P}(I-Q) N u(t) \\
&=K_{P} N u(t)-K_{P} Q N u(t) \\
&=\int_{0}^{1} G(t, s) N u(s) d s+l\left(I-\frac{1}{g(1)} g(B)\right) \varphi(N u) t \\
& \quad+\int_{0}^{1} G(t, s) d s \cdot Q N u(t)+l\left(I-\frac{1}{g(1)} g(B)\right) \varphi(Q N u) t \\
&=\int_{0}^{1} G(t, s) N u(s) d s+\frac{t(1-t)}{2} Q N u(t) \\
&+l\left(I-\frac{1}{g(1)} g(B)\right)(\varphi(N u)+\varphi(Q N u)) t .
\end{aligned}
$$

By using the standard argument, we can show that $Q N((\bar{\Omega}))$ is bounded and $K_{P}(I-$ Q) $N(\bar{\Omega})$ is compact. Thus, $N$ is $L$-compact on $\bar{\Omega}$.

The above results (Lemmas 2.1, 2.2, and 2.4) may be concrete for a specific matrix. In the following, we suppose that a diagonalizable matrix $B$ satisfy $B^{2}=I$ and $\operatorname{dim} \operatorname{Ker}(I-B)=k$. So, there exist a set of linearly independent vectors $\left\{\eta_{1}, \eta_{2}, \ldots, \eta_{n}\right\}$ such that

$$
B C=C\left(\begin{array}{cc}
I_{k} & 0 \\
0 & -I_{n-k}
\end{array}\right), \quad C=\left(\eta_{1}, \eta_{2}, \ldots, \eta_{n}\right), \eta_{i}=\left(\begin{array}{c}
\eta_{1 i} \\
\eta_{2 i} \\
\vdots \\
\eta_{n i}
\end{array}\right) \text {, }
$$

where $\eta_{i}(i=1,2, \ldots, k)$ is an eigenvector of $B$ with eigenvalue 1 and $\eta_{i}(i=k+1, k+2, \ldots, n)$ is an eigenvector of $B$ with eigenvalue -1 . Moreover, we shall suppose that

$$
\left(\begin{array}{cccc}
\eta_{11} & \eta_{12} & \cdots & \eta_{1 k} \\
\eta_{21} & \eta_{22} & \cdots & \eta_{2 k} \\
\vdots & \vdots & \cdots & \vdots \\
\eta_{k 1} & \eta_{k 2} & \cdots & \eta_{k k}
\end{array}\right)=I_{k}, \quad\left(\begin{array}{cccc}
\eta_{k+1, k+1} & \eta_{k+1, k+2} & \cdots & \eta_{k+1, n} \\
\eta_{k+2, k+1} & \eta_{k+2, k+2} & \cdots & \eta_{k+2, n} \\
\vdots & \vdots & \cdots & \vdots \\
\eta_{n, k+1} & \eta_{n, k+2} & \cdots & \eta_{n, n}
\end{array}\right)=I_{n-k} .
$$


Take $C^{-1}=\left(c_{i j}\right)_{n \times n}$,

$$
D_{1}=\left(\begin{array}{cccc}
c_{k+1,1} & c_{k+1,2} & \cdots & c_{k+1, k} \\
c_{k+2,1} & c_{k+2,2} & \cdots & c_{k+2, k} \\
\vdots & \vdots & \cdots & \vdots \\
c_{n, 1} & c_{n, 2} & \cdots & c_{n, k}
\end{array}\right), \quad D_{2}=\left(\begin{array}{cccc}
c_{k+1, k+1} & c_{k+1, k+2} & \cdots & c_{k+1, n} \\
c_{k+2, k+1} & c_{k+2, k+2} & \cdots & c_{k+2, n} \\
\vdots & \vdots & \cdots & \vdots \\
c_{n, k+1} & c_{n, k+2} & \cdots & c_{n, n}
\end{array}\right)
$$

It follows from $C^{-1} C=I$ and $(2.10)$ that, for $l, m \in\{1,2, \ldots, k\}$,

$$
\sum_{i=1}^{n} c_{l i} \eta_{i m}=\delta_{l m}= \begin{cases}1 & \text { if } l=m \\ 0 & \text { if } l \neq m\end{cases}
$$

Based on the notation above, (2.4) can be rewritten as

$$
C\left(\begin{array}{cc}
0 & 0 \\
0 & 2 I_{n-k}
\end{array}\right) C^{-1} \xi=\frac{1}{h} C\left(\begin{array}{cc}
I_{k} & 0 \\
0 & -I_{n-k}
\end{array}\right) C^{-1} \int_{0}^{1} \int_{0}^{1} G(t, s) \nu(s) d s d \alpha(t) .
$$

Thus,

$$
\left(\begin{array}{cc}
0 & 0 \\
0 & 2 I_{n-k}
\end{array}\right) C^{-1} \xi=\frac{1}{h}\left(\begin{array}{cc}
I_{k} & 0 \\
0 & -I_{n-k}
\end{array}\right) C^{-1} \int_{0}^{1} \int_{0}^{1} G(t, s) v(s) d s d \alpha(t) .
$$

Then the above matrix equation reduces to

$$
\left\{\begin{array}{l}
\int_{0}^{1} \int_{0}^{1} G(t, s) \sum_{j=1}^{n} c_{i j} v_{j}(s) d s d \alpha(t)=0, \quad i=1,2, \ldots, k, \\
-\int_{0}^{1} \int_{0}^{1} G(t, s) \sum_{j=1}^{n} c_{i j} v_{j}(s) d s d \alpha(t)=2 h \sum_{j=1}^{n} c_{i j} \xi_{j}, \quad i=k+1, k+2, \ldots, n,
\end{array}\right.
$$

or

$$
\left\{\begin{array}{l}
\int_{0}^{1} \int_{0}^{1} G(t, s) \sum_{j=1}^{n} c_{i j} v_{j}(s) d s d \alpha(t)=0, \quad i=1,2, \ldots, k \\
-\int_{0}^{1} \int_{0}^{1} G(t, s)\left(D_{1}, D_{2}\right) v(s) d s d \alpha(t)=2 h\left(D_{1}, D_{2}\right) \xi
\end{array}\right.
$$

Consequently,

$$
\operatorname{Im} L=\left\{v \in Y: \int_{0}^{1} \int_{0}^{1} G(t, s) \sum_{j=1}^{n} c_{i j} v_{j}(s) d s d \alpha(t)=0, i=1,2, \ldots, k\right\} .
$$

By (2.10), we know $\operatorname{det}\left(D_{2}\right) \neq 0$. From the second part of (2.12), we infer that

$$
\left(\begin{array}{c}
\xi_{k+1} \\
\xi_{k+2} \\
\vdots \\
\xi_{n}
\end{array}\right)=-\frac{D_{2}^{-1} D_{1}}{2 h}\left(\begin{array}{c}
\xi_{1} \\
\xi_{2} \\
\vdots \\
\xi_{k}
\end{array}\right)-\frac{1}{2 h} \int_{0}^{1} \int_{0}^{1} G(t, s)\left(D_{2}^{-1} D_{1}, I_{n-k}\right) v(s) d s d \alpha(t)
$$

Define the linear operator $P: X \rightarrow X$ by

$$
(P u)(t)=u_{1}(1) \eta_{1} t+u_{2}(1) \eta_{2} t+\cdots+u_{k}(1) \eta_{k} t, \quad u \in X,
$$


It follows from the left formula in (2.10) that $P$ is a continuous projection operator with $\operatorname{Im} P=\operatorname{Ker} L$.

Based on the assumption (H2), we define linear operators $Q: Y \rightarrow Y$ by

$$
Q v=\sum_{i=1}^{k} \gamma \int_{0}^{1} \int_{0}^{1} G(t, s) \sum_{j=1}^{n} c_{i j} v_{j}(s) d s d \alpha(t) \cdot \eta_{i}, \quad v \in Y,
$$

where $\gamma=\frac{2}{\int_{0}^{1} t(1-t) d \alpha(t)}$. For given $v \in Y$, we take

$$
\alpha_{i}=\int_{0}^{1} \int_{0}^{1} G(t, s) \sum_{j=1}^{n} c_{i j} v_{j}(s) d s d \alpha(t), \quad i=1,2, \ldots, k
$$

Then (2.13) reduces to

$$
(Q v)(t)=\gamma\left(\alpha_{1}, \alpha_{2}, \ldots, \alpha_{k}, \sum_{m=1}^{k} \alpha_{m} \eta_{k+1, m}, \sum_{m=1}^{k} \alpha_{m} \eta_{k+2, m}, \ldots, \sum_{m=1}^{k} \alpha_{m} \eta_{n, m}\right) .
$$

With the help of (2.11), we have

$$
\begin{aligned}
\frac{1}{\gamma} \sum_{j=1}^{n} c_{i j}(Q v)_{j}(s) \\
=c_{i 1} \alpha_{1}+c_{i 2} \alpha_{2}+\cdots+c_{i k} \alpha_{k}+c_{i k+1} \sum_{m=1}^{k} \alpha_{m} \eta_{k+1, m} \\
\quad+c_{i k+2} \sum_{m=1}^{k} \alpha_{m} \eta_{k+2, m}+\cdots+c_{i n} \sum_{m=1}^{k} \alpha_{m} \eta_{n, m} \\
=\alpha_{1}\left(c_{i 1}+c_{i k+1} \eta_{k+1,1}+c_{i k+2} \eta_{k+2,1}+\cdots+c_{i n} \eta_{n, 1}\right) \\
\quad+\alpha_{2}\left(c_{i 2}+c_{i k+1} \eta_{k+1,2}+c_{i k+2} \eta_{k+2,2}+\cdots+c_{i n} \eta_{n, 2}\right) \\
\quad+\cdots+\alpha_{k}\left(c_{i k}+c_{i k+1} \eta_{k+1, k}+c_{i k+2} \eta_{k+2, k}+\cdots+c_{i n} \eta_{n, k}\right) \\
=\alpha_{1} \delta_{i 1}+\alpha_{2} \delta_{i 2}+\cdots+\alpha_{k} \delta_{i k}, \quad i=, 1,2, \ldots, k .
\end{aligned}
$$

Consequently,

$$
\begin{aligned}
\left(Q^{2} v\right)(t) & =\sum_{i=1}^{k} \gamma \int_{0}^{1} \int_{0}^{1} G(t, s) \sum_{j=1}^{n} c_{i j}(Q v)_{j}(s) d s d \alpha(t) \cdot \eta_{i} \\
& =\gamma \int_{0}^{1} \int_{0}^{1} G(t, s) d s d \alpha(t)\left(\sum_{i=1}^{k} \gamma \int_{0}^{1} \int_{0}^{1} G(t, s) \sum_{j=1}^{n} c_{i j} \nu_{j}(s) d s d \alpha(t) \cdot \eta_{i}\right) \\
& =\gamma \int_{0}^{1} \frac{t(1-t)}{2} d \alpha(t)\left(\sum_{i=1}^{k} \gamma \int_{0}^{1} \int_{0}^{1} G(t, s) \sum_{j=1}^{n} c_{i j} \nu_{j}(s) d s d \alpha(t) \cdot \eta_{i}\right) \\
& =(Q v)(t) .
\end{aligned}
$$


This implies that $Q$ is a continuous projection operator. Obviously, $\operatorname{Ker} Q=\operatorname{Im} L$ holds from the linear independence of the vectors $\left\{\eta_{1}, \eta_{2}, \ldots, \eta_{k}\right\}$.

From (2.12), it follows that the generalized inverse $K_{P}: \operatorname{Im} L \rightarrow \operatorname{dom} L \cap \operatorname{Ker} P$ can be defined by

$$
\left(K_{P} v\right)(t)=\int_{0}^{1} G(t, s) v(s) d s+\delta \cdot t
$$

where $\delta \in \mathbb{R}^{n}$ is given by

$$
\delta=(\underbrace{0, \ldots, 0}_{k}, \frac{1}{2 h} \int_{0}^{1} \int_{0}^{1} G(t, s)\left(D_{2}^{-1} D_{1}, I_{n-k}\right) v(s) d s d \alpha(t))^{T} .
$$

Similar to the proof of Lemma 2.4, we obtain

$$
\left\|K_{P} v\right\|_{X} \leq\left(1+\frac{n}{2 h}\left\|\left(D_{2}^{-1} D_{1}, I_{n-k}\right)\right\|_{*} \int_{0}^{1} t(1-t) d\left(\bigvee_{0}^{t}(\alpha)\right)\right)\|v\|_{1} .
$$

\section{Main result}

In this section, we use Theorem 1.1 to prove the existence of solutions to (1.1). For this purpose, we use the following assumptions:

(H5) There exist nonnegative functions $a, b, c \in L^{1}[0,1]$ such that, for all $u, v \in \mathbb{R}^{n}$ and $t \in[0,1]$,

$$
|f(t, u, v)| \leq a(t)\|u\|_{\mathbb{R}^{n}}+b(t)\|v\|_{\mathbb{R}^{n}}+c(t) .
$$

(H6) There exists a constant $\Lambda>0$ such that, for each $u \in \operatorname{dom} L$, if $\left\|u^{\prime}(t)\right\|_{\mathbb{R}^{n}}>\Lambda$ for all $t \in[0,1]$, then

$$
g(B) A \int_{0}^{1} \int_{0}^{1} G(t, s) f\left(s, u(s), u^{\prime}(s)\right) d s d \alpha(t) \neq 0 .
$$

(H7) There exists a constant $\Lambda_{1}>0$ such that either for any $\psi \in \mathbb{R}^{n}$ with $\psi=B \psi$ and $\|\psi\|_{\mathbb{R}^{n}}>\Lambda_{1}$

$$
(\psi, Q N u) \leq 0,
$$

or for any $\psi \in \mathbb{R}^{n}$ with $\psi=B \psi$ and $\|\psi\|_{\mathbb{R}^{n}}>\Lambda_{1}$,

$$
(\psi, Q N u) \geq 0,
$$

where $u(t)=\psi t$, and $(\cdot, \cdot)$ denotes the scalar product in $\mathbb{R}^{n}$.

Theorem 3.1 Let the assumptions $(H 1)-(H 7)$ hold. Then (1.1) has at least one solution in $X$ provided that $\left(n\|g(B)\|_{*}+M|g(1)|\right)\left(\|a\|_{1}+\|b\|_{1}\right)<|g(1)|$.

Proof Set

$$
\Omega_{1}=\{u \in \operatorname{dom} L \backslash \operatorname{Ker} L: L u=\lambda N u \text { for some } \lambda \in[0,1]\} .
$$


Suppose that $u \in \Omega_{1}$, and $L u=\lambda N u$. Then $\lambda \neq 0$ and $N u \in \operatorname{Im} L=\operatorname{Ker} Q$ so that

$$
g(B) A \int_{0}^{1} \int_{0}^{1} G(t, s) f\left(s, u(t), u^{\prime}(t)\right)=0, \quad \text { for all } t \in[0,1] .
$$

Thus, from (H6), there is $t_{0} \in[0,1]$ such that $\left\|u^{\prime}\left(t_{0}\right)\right\|_{\mathbb{R}^{n}} \leq \Lambda$. By the absolute continuity of $u$, for $t \in[0,1]$, we have

$$
|u(t)|=\left|u(0)-\int_{0}^{t} u^{\prime}(s) d s\right| \leq\left\|u^{\prime}\right\|_{\infty}
$$

and

$$
\left|u^{\prime}(t)\right|=\left|u^{\prime}\left(t_{0}\right)-\int_{t_{0}}^{t} u^{\prime \prime}(s) d s\right| \leq \Lambda+\left\|u^{\prime \prime}\right\|_{1} .
$$

This yields

$$
\|P u\|_{X}=\max \left\{\|P u\|_{\infty},\left\|(P u)^{\prime}\right\|_{\infty}\right\} \leq \frac{n\|g(B)\|_{*}}{|g(1)|}\|u(1)\|_{\mathbb{R}^{n}} \leq \frac{n\|g(B)\|_{*}}{|g(1)|}\left\|u^{\prime}\right\|_{\infty} .
$$

Again, if $u \in \operatorname{dom} L$, then $(I-P) u \in \operatorname{dom} L \cap \operatorname{Ker} P$ and $L P u=0$. Then, by (2.7) and Lemma 2.3,

$$
\begin{aligned}
\|(I-P) u\|_{X} & =\left\|K_{P} L(I-P) u\right\|_{X} \leq M\|L(I-P) u\|_{1} \\
& =M\|L u\|_{1}=M\left\|u^{\prime \prime}\right\|_{1} \leq M\|N u\|_{1} .
\end{aligned}
$$

Using (3.3), (3.4) and (3.5), we conclude that

$$
\begin{aligned}
\|u\|_{X} & =\|P u+(I-P) u\|_{X} \leq\|P u\|_{X}+\|(I-P) u\|_{X} \\
& \leq \frac{n\|g(B)\|_{*}}{|g(1)|}\left(\Lambda+\left\|u^{\prime \prime}\right\|_{1}\right)+M\left\|u^{\prime \prime}\right\|_{1} \\
& =\frac{n\|g(B)\|_{*}}{|g(1)|} \Lambda+\left(\frac{n\|g(B)\|_{*}}{|g(1)|}+M\right)\left\|u^{\prime \prime}\right\|_{1} \\
& \leq \frac{n\|g(B)\|_{*}}{|g(1)|} \Lambda+\left(\frac{n\|g(B)\|_{*}}{|g(1)|}+M\right) \int_{0}^{1}\left|f\left(s, u(s), u^{\prime}(s)\right)\right| d s \\
& \leq \frac{n\|g(B)\|_{*}}{|g(1)|} \Lambda+\left(\frac{n\|g(B)\|_{*}}{|g(1)|}+M\right)\left(\|a\|_{1}\|u\|_{\infty}+\|b\|_{1}\left\|u^{\prime}\right\|_{\infty}+\|c\|_{1}\right) \\
& \leq \frac{n\|g(B)\|_{*}}{|g(1)|} \Lambda+\left(\frac{n\|g(B)\|_{*}}{|g(1)|}+M\right)\|c\|_{1}+\left(\frac{n\|g(B)\|_{*}}{|g(1)|}+M\right)\left(\|a\|_{1}+\|b\|_{1}\right)\|u\|_{X} .
\end{aligned}
$$

The last inequality allows us to deduce that

$$
\|u\|_{X} \leq \frac{n\|g(B)\|_{*} \Lambda+\left(n\|g(B)\|_{*}+M|g(1)|\right)\|c\|_{1}}{|g(1)|-\left(n\|g(B)\|_{*}+M|g(1)|\right)\left(\|a\|_{1}+\|b\|_{1}\right)} .
$$

Thus, $\Omega_{1}$ is bounded. Let

$$
\Omega_{2}=\{u \in \operatorname{Ker} L: N u \in \operatorname{Im} L\} .
$$


For $u \in \Omega_{2}$ and from the definition of $\operatorname{Im} L, u(t)=\psi t$, where $\psi \in \mathbb{R}^{n}$. Since $Q N u=0$, we have

$$
g(B) A \int_{0}^{1} \int_{0}^{1} G(t, s) f\left(s, u(s), u^{\prime}(s)\right) d s d \alpha(t)=0 .
$$

Hence, from (H6), we can show that

$$
\|u\|_{X}=\|u\|_{\infty}=\|\psi\|_{\mathbb{R}^{n}} \leq \Lambda .
$$

Therefore, $\Omega_{2}$ is a bounded set in $X$.

Let $J(\psi)=\psi t$ be the isomorphism. Then we want to show that the set of $u$ in $\operatorname{Ker} L$ such that

$$
-\lambda u+(1-\lambda) J Q N u=0
$$

with $\lambda \in[0,1]$ is bounded if (3.1) holds. This means that (with $\psi=B \psi$ and $u=\psi t$ )

$$
-\lambda \psi t+(1-\lambda) Q N(\psi t) t=0,
$$

or

$$
-\lambda \psi+(1-\lambda) Q N(\psi t)=0 .
$$

If $\lambda=0$, we have $Q N(\psi t)=0$, that is,

$$
g(B) A \int_{0}^{1} \int_{0}^{1} G(t, s) f(s, \psi s, \psi) d s d \alpha(t)=0 .
$$

Thus, we deduce that $\|\psi\|_{\mathbb{R}^{n}} \leq \Lambda$ follows from (H5). Otherwise, if $\|\psi\|_{\mathbb{R}^{n}}>\Lambda_{1}$, in view of (H7), we have

$$
0<\lambda\|\psi\|_{\mathbb{R}^{n}}^{2}=(1-\lambda)(\psi, Q N(\psi t)) \leq 0 .
$$

Thus, $\|u\|_{X}=\|\psi\|_{\mathbb{R}^{n}} \leq \Lambda_{1}$. Using the same argument as above, we can conclude that the set of $u$ in $\operatorname{Ker} L$ such that

$$
\lambda u+(1-\lambda) J Q N u=0
$$

with $\lambda \in[0,1]$ is bounded if (3.2) holds. Therefore, the set

$$
\Omega_{3}=\{u \in \operatorname{Ker} L: \mu \lambda u+(1-\lambda) J Q N u=0, \lambda \in[0,1]\}
$$

is bounded if conditions (H6) and (H7) are satisfied, where

$$
\mu= \begin{cases}-1 & \text { if (3.1) holds; } \\ 1 & \text { if (3.2) holds }\end{cases}
$$


Finally, the proof of this theorem is now an easy consequence of Lemmas 2.1, 2.2, and 2.3 and Theorem 1.1. Let $\Omega$ be a bounded and open subset of $X \bigcup_{i=1}^{3} \overline{\Omega_{i}} \subset \Omega$. Then, by the above argument, we have

(i) $L x \neq \lambda N x$, for every $(x, \lambda) \in[(\operatorname{dom} L \backslash \operatorname{Ker} L) \cap \partial \Omega] \times(0,1)$,

(ii) $N x \notin \operatorname{Im} L$ for $x \in \operatorname{Ker} L \cap \partial \Omega$,

(iii) $H(x, \lambda)=\mu \lambda x+(1-\lambda) J Q N x$. By the homotopy property of degree,

$$
\operatorname{deg}\left(\left.J Q N\right|_{\operatorname{Ker} L}, \operatorname{Ker} L \cap \Omega, 0\right)=\operatorname{deg}(\mu I, \operatorname{Ker} L \cap \Omega, 0) \neq 0 .
$$

Then, by Theorem 1.1, $L u=N u$ has at least one solution in $\operatorname{dom} L \cap \bar{\Omega}$ so that the IBVP (1.1) has at least one solution.

For the special case that a diagonalizable matrix $B$ satisfy $B^{2}=I$ and $\operatorname{dim} \operatorname{Ker}(I-B)=k$, we make the following assumptions:

(H8) There exists a constant $\Lambda>0$ such that, for each $u \in \operatorname{dom} L$, if $\left|u_{1}^{\prime}(t)\right|>\Lambda$ for all $t \in[0,1]$ or $\left|u_{2}^{\prime}(t)\right|>\Lambda$ for all $t \in[0,1]$, or $\ldots$, or $\left|u_{k}^{\prime}(t)\right|>\Lambda$ for all $t \in[0,1]$, then

$$
(Q N u)(t)=\sum_{i=1}^{k} \gamma \int_{0}^{1} \int_{0}^{1} G(t, s) \sum_{j=1}^{n} c_{i j} f_{j}\left(s, u(s), u^{\prime}(s)\right) d s d \alpha(t) \cdot \eta_{i} \neq 0 .
$$

Theorem 3.2 Let the assumptions (H2), (H3), (H5), (H7), and (H8) hold. Then (1.1) has at least one solution in $X$ provided that $\left(\left\|\sum_{i=1}^{k} \eta_{i}\right\|_{\mathbb{R}^{n}}+M_{1}\right)\left(\|a\|_{1}+\|b\|_{1}\right)<1$, where $M_{1}=$ $\left(1+\frac{n}{2 h}\left\|\left(D_{2}^{-1} D_{1}, I_{n-k}\right)\right\|_{*} \int_{0}^{1} t(1-t) d\left(\bigvee_{0}^{t}(\alpha)\right)\right)$.

Proof For $u \in \Omega_{1}, Q N u=0$. Then, from (H8), there is $t_{i} \in[0,1](i=1,2, \ldots, k)$ such that $\left|u_{i}^{\prime}\left(t_{i}\right)\right| \leq \Lambda$. By the absolute continuity of $u_{i}$, for $t \in[0,1]$, we have

$$
\left|u_{i}(t)\right|=\left|u_{i}(0)-\int_{0}^{t} u_{i}^{\prime}(s) d s\right| \leq\left\|u^{\prime}\right\|_{\infty}, \quad i=1,2, \ldots, k
$$

and

$$
\left|u_{i}^{\prime}(t)\right|=\left|u_{i}^{\prime}\left(t_{i}\right)-\int_{t_{i}}^{t} u_{i}^{\prime \prime}(s) d s\right| \leq \Lambda+\left\|u^{\prime \prime}\right\|_{1}, \quad i=1,2, \ldots, k
$$

This yields

$$
\begin{aligned}
\|P u\|_{X} & =\max \left\{\|P u\|_{\infty},\left\|(P u)^{\prime}\right\|_{\infty}\right\}=\|P u\|_{\infty} \\
& \leq \max _{1 \leq i \leq k}\left\{\left|u_{i}(1)\right|\right\} \cdot\left\|\sum_{i=1}^{k} \eta_{i}\right\|_{\mathbb{R}^{n}} \leq\left\|\sum_{i=1}^{k} \eta_{i}\right\|_{\mathbb{R}^{n}} \cdot\left\|u^{\prime}\right\|_{\infty} .
\end{aligned}
$$

Again, if $u \in \operatorname{dom} L$, then $(I-P) u \in \operatorname{dom} L \cap \operatorname{Ker} P$ and $L P u=0$. Then, by (2.15),

$$
\begin{aligned}
\|(I-P) u\|_{X} & =\left\|K_{P} L(I-P) u\right\|_{X} \leq M_{1}\|L(I-P) u\|_{1} \\
& =M_{1}\|L u\|_{1}=M_{1}\left\|u^{\prime \prime}\right\|_{1} \leq M_{1}\|N u\|_{1} .
\end{aligned}
$$


Using the above three inequalities, we conclude that

$$
\begin{aligned}
\|u\|_{X} & =\|P u+(I-P) u\|_{X} \leq\|P u\|_{X}+\|(I-P) u\|_{X} \\
& \leq\left\|\sum_{i=1}^{k} \eta_{i}\right\|_{\mathbb{R}^{n}}\left(\Lambda+\left\|u^{\prime \prime}\right\|_{1}\right)+M_{1}\left\|u^{\prime \prime}\right\|_{1}=\left\|\sum_{i=1}^{k} \eta_{i}\right\|_{\mathbb{R}^{n}} \Lambda \\
& +\left(\left\|\sum_{i=1}^{k} \eta_{i}\right\|_{\mathbb{R}^{n}}+M_{1}\right)\left\|u^{\prime \prime}\right\|_{1} \quad \\
& \leq\left\|\sum_{i=1}^{k} \eta_{i}\right\|_{\mathbb{R}^{n}} \Lambda+\left(\left\|\sum_{i=1}^{k} \eta_{i}\right\|_{\mathbb{R}^{n}}+M_{1}\right) \int_{0}^{1}\left|f\left(s, u(s), u^{\prime}(s)\right)\right| d s \\
& \leq\left\|\sum_{i=1}^{k} \eta_{i}\right\|_{\mathbb{R}^{n}} \Lambda+\left(\left\|\sum_{i=1}^{k} \eta_{i}\right\|_{\mathbb{R}^{n}}+M_{1}\right)\|c\|_{1} \\
& +\left(\left\|\sum_{i=1}^{k} \eta_{i}+M_{\mathbb{R}^{n}}\left(\|a\|_{1}+\|b\|_{1}\right)\right\| u \|_{X} .\right.
\end{aligned}
$$

The last inequality allows us to deduce that

$$
\|u\|_{X} \leq \frac{\left\|\sum_{i=1}^{k} \eta_{i}\right\|_{\mathbb{R}^{n}} \Lambda+\left(\left\|\sum_{i=1}^{k} \eta_{i}\right\|_{\mathbb{R}^{n}}+M_{1}\right)\|c\|_{1}}{1-\left(\left\|\sum_{i=1}^{k} \eta_{i}\right\|_{\mathbb{R}^{n}}+M_{1}\right)\left(\|a\|_{1}+\|b\|_{1}\right)} .
$$

Thus, $\Omega_{1}$ is bounded. The rest of the proof repeats that of Theorem 3.1.

Example 3.1 Consider the differential system

$$
\left\{\begin{array}{l}
-x_{1}^{\prime \prime}(t)=\sin x_{2}(t) x_{2}^{\prime}(t)+\frac{1}{5} \arctan x_{2}(t)+\frac{1}{5} x_{1}^{\prime}(t)+t, \\
-x_{2}^{\prime \prime}(t)=\sin x_{1}(t) x_{3}(t)-\cos x_{3}^{\prime}(t)+\frac{1}{20} x_{2}^{\prime}(t)-\frac{1}{20} x_{2}(t)+e^{t}, \\
-x_{3}^{\prime \prime}(t)=\arctan x_{3}^{2}(t)+\cos x_{1}(t)+\cos x_{2}(t)+\frac{1}{20} x_{2}(t)+\frac{1}{10} x_{2}^{\prime}(t), \\
x_{1}(0)=x_{2}(0)=x_{3}(0)=0, \quad x_{1}(1)=2 \int_{0}^{1} x_{1}(t) d t, \\
x_{2}(1)=2 \int_{0}^{1} x_{3}(t) d t, \quad x_{3}(1)=2 \int_{0}^{1} x_{2}(t) d t .
\end{array}\right.
$$

Here $f_{i}:[0,1] \times \mathbb{R}^{6} \rightarrow \mathbb{R}, i=1,2,3$ are defined, respectively, by

$$
\begin{aligned}
& f_{1}(t, x, y)=\sin x_{2} y_{2}+\frac{1}{5} \arctan x_{2}+\frac{1}{5} y_{1}+t, \\
& f_{2}(t, x, y)=\sin x_{1} x_{3}-\cos y_{3}-\frac{1}{20} y_{2}-\frac{1}{20} x_{2}+e^{t}, \\
& f_{3}(t, x, y)=\arctan x_{3}^{2}+\cos x_{1}+\cos x_{2}+\frac{1}{20} x_{2}+\frac{1}{10} y_{2},
\end{aligned}
$$

where $x=\left(x_{1}, x_{2}, x_{3}\right)^{T}, y=\left(y_{1}, y_{2}, y_{3}\right)^{T} \in \mathbb{R}^{3}$.

Take $\alpha(t)=t$,

$$
A=\left(\begin{array}{lll}
2 & 0 & 0 \\
0 & 0 & 2 \\
0 & 2 & 0
\end{array}\right)
$$


and

$$
f(t, x, y)=\left(\begin{array}{l}
f_{1}(t, x, y) \\
f_{2}(t, x, y) \\
f_{3}(t, x, y)
\end{array}\right) .
$$

Then $h=\int_{0}^{1} t d \alpha(t)=\frac{1}{2}, B=A h=\frac{1}{2} A, B^{2}=I$,

$$
\begin{aligned}
& C=\left(\begin{array}{ccc}
1 & 0 & 0 \\
0 & 1 & -1 \\
0 & 1 & 1
\end{array}\right)=\left(\eta_{1}, \eta_{2}, \eta_{3}\right), \quad C^{-1}=\left(\begin{array}{ccc}
1 & 0 & 0 \\
0 & \frac{1}{2} & \frac{1}{2} \\
0 & -\frac{1}{2} & \frac{1}{2}
\end{array}\right)=\left(c_{i j}\right)_{3 \times 3}, \\
& D_{2}=\frac{1}{2}, \quad D_{1}=\left(0,-\frac{1}{2}\right), \quad n=3, \quad k=2, \\
&(Q v)(t)=12 \int_{0}^{1} \int_{0}^{1} G(t, s) v_{1}(s) d s d \alpha(t) \cdot \eta_{1} \\
&+6 \int_{0}^{1} \int_{0}^{1} G(t, s)\left(v_{2}(s)+v_{3}(s)\right) d s d \alpha(t) \cdot \eta_{2} \\
&=\left(\begin{array}{c}
12 \int_{0}^{1} \int_{0}^{1} G(t, s) v_{1}(s) d s d \alpha(t) \\
6 \int_{0}^{1} \int_{0}^{1} G(t, s)\left(v_{2}(s)+v_{3}(s)\right) d s d \alpha(t) \\
6 \int_{0}^{1} \int_{0}^{1} G(t, s)\left(v_{2}(s)+v_{3}(s)\right) d s d \alpha(t)
\end{array}\right) .
\end{aligned}
$$

It follows from (3.7) that

$$
|f(t, x, y)| \leq \frac{1}{20}|x|+\frac{1}{5}|y|+4
$$

Note that $M_{1}=\left(1+\frac{n}{2 h}\left\|\left(D_{2}^{-1} D_{1}, I_{n-k}\right)\right\|_{*} \int_{0}^{1} t(1-t) d\left(\bigvee_{0}^{t}(\alpha)\right)\right)$, we have $M_{1}=\frac{3}{2}$. Then we obtain $\left(\left\|\sum_{i=1}^{2} \eta_{i}\right\|_{\mathbb{R}^{3}}+M_{1}\right)\left(\|a\|_{1}+\|b\|_{1}\right)=\frac{5}{8}<1$. Therefore, condition (H5) is satisfied.

Take $\Lambda=200$. Then, for $\left|y_{1}(t)\right| \geq \Lambda$ for all $t \in[0,1]$, we have

$$
\begin{aligned}
& \left|f_{1}(t, x, y)\right| \\
& \quad=\left|\sin x_{2} y_{2}+\frac{1}{5} \arctan x_{2}+\frac{1}{5} y_{1}+t\right| \geq \frac{1}{5}\left|y_{1}\right|-4 \geq 36
\end{aligned}
$$

and for $\left|y_{2}(t)\right| \geq \Lambda$ for all $t \in[0,1]$, we have

$$
\begin{aligned}
& \left|\left(f_{2}+f_{3}\right)(t, x, y)\right| \\
& \quad=\left|\sin x_{1} x_{3}-\cos y_{3}+e^{t}+\arctan x_{3}^{2}+\cos x_{1}+\cos x_{2}+\frac{1}{10} y_{2}\right| \geq \frac{1}{20}\left|y_{2}\right|-6 \geq 4 .
\end{aligned}
$$

Thus, for each $u \in X$, if $\left|v_{1}(t)\right| \geq \Lambda$ for all $t \in[0,1]$ or $\left|v_{2}(t)\right| \geq \Lambda$ for all $t \in[0,1]$, we conclude that

$$
(Q N v)(t)=\left(\begin{array}{c}
12 \int_{0}^{1} \int_{0}^{1} G(t, s) v_{1}(s) d s d \alpha(t) \\
6 \int_{0}^{1} \int_{0}^{1} G(t, s)\left(v_{2}(s)+v_{3}(s)\right) d s d \alpha(t) \\
6 \int_{0}^{1} \int_{0}^{1} G(t, s)\left(v_{2}(s)+v_{3}(s)\right) d s d \alpha(t)
\end{array}\right) \neq 0 .
$$

Hence, (H8) holds. 
Let $\psi=\left(c_{1}, c_{2}, c_{2}\right)^{T} \in \operatorname{Ker}(I-B)=\left\{c_{1}(1,0,0)+c_{2}(0,1,1): c_{1}, c_{2} \in \mathbb{R}\right\}$. Then we have

$$
\begin{aligned}
& f_{1}(t, \psi t, \psi)=\sin c_{2}^{2} t+\frac{1}{5} \arctan c_{2} t+\frac{1}{5} c_{1}+t \\
& f_{2}(t, \psi t, \psi)=\sin c_{1} c_{2} t^{2}-\cos c_{2}-\frac{1}{20} c_{2}-\frac{t}{20} c_{2}+e^{t} \\
& f_{3}(t, \psi t, \psi)=\arctan c_{2}^{2} t^{2}+\cos c_{1} t+\cos c_{2} t+\frac{t}{20} c_{2}+\frac{1}{10} c_{2}
\end{aligned}
$$

and

$$
\begin{aligned}
c_{1} f_{1}(t, \psi t, \psi)+c_{2}\left(f_{2}(t, \psi t, \psi)+f_{3}(t, \psi t, \psi)\right) \\
=c_{1}\left(\sin c_{2}^{2} t+\frac{1}{5} \arctan c_{2} t+\frac{1}{5} c_{1}+t\right) \\
\quad+c_{2}\left(\frac{1}{10} c_{2}+\sin c_{1} c_{2} t^{2}-\cos c_{2}+e^{t}+\arctan c_{2}^{2} t^{2}+\cos c_{1} t+\cos c_{2} t\right) \\
\geq \frac{1}{5} c_{1}^{2}-3\left|c_{1}\right|+\frac{1}{10} c_{2}^{2}-10\left|c_{2}\right| \geq \frac{1}{10} \max \left\{c_{1}^{2}, c_{2}^{2}\right\}-13 \max \left\{\left|c_{1}\right|,\left|c_{2}\right|\right\} .
\end{aligned}
$$

Note that $\|\psi\|_{\mathbb{R}^{3}}=\max \left\{\left|c_{1}\right|,\left|c_{2}\right|\right\}$ and

$$
(\psi, Q N(\psi t))=12 \int_{0}^{1} \int_{0}^{1} G(t, s)\left[c_{1} f_{1}+c_{2}\left(f_{2}+f_{3}\right)\right] d s d t \geq 156, \quad \text { if }\|\psi\|_{\mathbb{R}^{3}} \geq 131
$$

we see that condition (H7) is satisfied. It follows from Theorem 3.2 that the problem (3.7) has at least one solution.

\section{Acknowledgements}

The authors wish to thank the anonymous referees for their valuable suggestions.

\section{Funding}

This work was partially supported by the Natural Science Foundation of China $(11371221,11571207,51774197)$, the Shandong Natural Science Foundation (ZR2018MA011), SDUST graduate innovation project (SDKDYC190238), and the Tai'shan Scholar Engineering Construction Fund of Shandong Province of China.

\section{Availability of data and materials}

Not applicable.

\section{Competing interests}

The authors declare that they have no competing interests.

\section{Authors' contributions}

The authors have equally made the contributions. All authors read and approved the final manuscript.

\section{Author details}

${ }^{1}$ Department of Applied Mathematics, Shandong University of Science and Technology, Qingdao, P.R. China. ${ }^{2}$ State Key Laboratory of Mining Disaster Prevention and Control Co-founded by Shandong Province and the Ministry of Science and Technology, Shandong University of Science and Technology, Qingdao, P.R. China.

\section{Publisher's Note}

Springer Nature remains neutral with regard to jurisdictional claims in published maps and institutional affiliations.

Received: 29 January 2019 Accepted: 10 September 2019 Published online: 23 September 2019

\section{References}

1. Asif, N.A., Khan, R.A.: Positive solutions to singular system with four-point coupled boundary conditions. J. Math. Anal. Appl. 386, 848-861 (2012) 
2. Bai, Z:: On solutions of some fractional $m$-point boundary value problems at resonance. Electron. J. Qual. Theory Differ. Equ. 37, 1 (2010)

3. Cui, Y: Solvability of second-order boundary value problems at resonance involving integral conditions. Electron. J. Differ. Equ. 2012, 45 (2012)

4. Cui, Y.: Uniqueness of solution for boundary value problems for fractional differential equations. Appl. Math. Lett. 51, 48-54 (2016)

5. Cui, Y., Liu, L., Zhang, X.: Uniqueness and existence of positive solutions for singular differential systems with coupled integral boundary value problems. Abstr. Appl. Anal. 2013, Article ID 340487 (2013)

6. Cui, Y., Ma, W., Wang, X., Su, X.: Uniqueness theorem of differential system with coupled integral boundary conditions. Electron. J. Qual. Theory Differ. Equ. 2018, 9 (2018)

7. Cui, Y., Sun, J.: On existence of positive solutions of coupled integral boundary value problems for a nonlinear singular superlinear differential system. Electron. J. Qual. Theory Differ. Equ. 2012, 41 (2012)

8. Cui, Y., Zou, Y.: Monotone iterative method for differential systems with coupled integral boundary value problems. Bound. Value Probl. 2013, 245 (2013)

9. Cui, Y., Zou, Y.: An existence and uniqueness theorem for a second order nonlinear system with coupled integral boundary value conditions. Appl. Math. Comput. 256, 438-444 (2015)

10. Cui, Y., Zou, Y.: Existence of solutions for second-order integral boundary value problems. Nonlinear Anal., Model. Control 21(6), 828-838 (2016)

11. Henderson, J., Luca, R., Tudorache, A.: On a system of fractional differential equations with coupled integral boundary conditions. Fract. Calc. Appl. Anal. 18, 361-386 (2015)

12. Infante, G., Minhós, F., Pietramala, P.: Non-negative solutions of systems of ODEs with coupled boundary conditions. Commun. Nonlinear Sci. Numer. Simul. 17, 4952-4960 (2012)

13. Jiang, J., Liu, L., Wu, Y.: Symmetric positive solutions to singular system with multi-point coupled boundary conditions. Appl. Math. Comput. 220(4), 536-548 (2013)

14. Jiang, J., Liu, L., Wu, Y.: Positive solutions to singular fractional differential system with coupled boundary conditions. Commun. Nonlinear Sci. Numer. Simul. 18(11), 3061-3074 (2013)

15. Jiang, J., Liu, L., Wu, Y.: Positive solutions for second-order differential equations with integral boundary conditions. Bull. Malays. Math. Sci. Soc. 37(3), 779-796 (2014)

16. Kosmatov, N.: A singular non-local problem at resonance. J. Math. Anal. Appl. 394, 425-431 (2012)

17. Li, H., Zhang, J.: Global structure of positive solutions for some second order multi-point boundary value problems. J. Funct. Spaces 2017, Article ID 1014250 (2017)

18. Ma, W., Meng, S., Cui, Y.: Resonant integral boundary value problems for Caputo fractional differential equations. Math. Probl. Eng. 2018, Article ID 5438592 (2018)

19. Mawhin, J.: Topological Degree Methods in Nonlinear Boundary-Value Problems. Nsfcbms Regional Conference Series in Mathematics. American Mathematical Society, Providence (1979)

20. Meng, S., Cui, Y:: Multiplicity results to a conformable fractional differential equations involving integral boundary condition. Complexity 2019, Article ID 8402347 (2019)

21. Meng, S., Cui, Y.: The extremal solution to conformable fractional differential equations involving integral boundary condition. Mathematics 7, 186 (2019). https://doi.org/10.3390/math7020186

22. Phung, P.D., Truong, L.X.: On the existence of a three point boundary value problem at resonance in $\mathbb{R}^{n}$. J. Math. Anal. Appl. 416, 522-533 (2014)

23. Qi, T., Liu, Y., Cui, Y.: Existence of solutions for a class of coupled fractional differential systems with nonlocal boundary conditions. J. Funct. Spaces 2017, Article ID 6703860 (2017)

24. Qi, T., Liu, Y., Zou, Y.: Existence result for a class of coupled fractional differential systems with integral boundary value conditions. J. Nonlinear Sci. Appl. 10, 4034-4045 (2017)

25. Song, F., Yang, H.: Modeling and analysis of fractional neutral disturbance waves in arterial vessels. Math. Model. Nat. Phenom. 14, 301 (2019)

26. Sun, F., Liu, L., Zhang, X., Wu, Y.: Spectral analysis for a singular differential system with integral boundary conditions. Mediterr. J. Math. 13, 4763-4782 (2016)

27. Sun, Q., Cui, Y.: Existence results for $(k, n-k)$ conjugate boundary-value problems with integral boundary conditions at resonance with $\operatorname{dim} \operatorname{ker} L=2$. Bound. Value Probl. 2017, 69 (2017)

28. Sun, Q., Meng, S., Cui, Y.: Existence results for fractional order differential equation with nonlocal Erdélyi-Kober and generalized Riemann-Liouville type integral boundary conditions at resonance. Adv. Differ. Equ. 2018, 243 (2018)

29. Truong, L.X., Phung, P.D.: Existence of positive solutions for a multi-point fourth order boundary value problem. Electron. J. Differ. Equ. 2011, 129 (2011)

30. Truong, L.X., Phung, P.D., Quan, B.T.: Positive pseudo-symmetric solutions for a nonlocal p-Laplacian boundary value problem. Differ. Equ. Appl. 5(1), 53-68 (2013)

31. Wang, Y., Liu, L., Wu, Y.: Positive solutions for a class of higher-order singular semipositone fractional differential system. Adv. Differ. Equ. 2014, 268 (2014)

32. Wang, Y., Liu, L., Zhang, X., Wu, Y.: Positive solutions of a fractional semipositone differential system arising from the study of HIV infection models. Appl. Math. Comput. 258, 312-324 (2015)

33. Yue, Z., Zou, Y.: New uniqueness results for fractional differential equation with dependence on the first order derivative. Adv. Differ. Equ. 2019, 38 (2019)

34. Zhai, C., Wang, W., Li, H.: A uniqueness method to a new Hadamard fractional differential system with four-point boundary conditions. J. Inequal. Appl. 2018, 207 (2018)

35. Zhang, J., Zhang, G., Li, H.: Positive solutions of second-order problem with dependence on derivative in nonlinearity under Stieltjes integral boundary condition. Electron. J. Qual. Theory Differ. Equ. 2018, 4 (2018)

36. Zhang, Y.: Existence results for a coupled system of nonlinear fractional multi-point boundary value problems at resonance. J. Inequal. Appl. 2018, 198 (2018)

37. Zou, Y., He, G.: On the uniqueness of solutions for a class of fractional differential equations. Appl. Math. Lett. 74, 68-73 (2017) 